\title{
SYMMETRIZATION AND OPTIMAL CONTROL FOR ELLIPTIC EQUATIONS
}

\author{
CHARLES VOAS AND DANIEL YANIRO
}

\begin{abstract}
We consider an optimal control problem where $u(x)$ satisfies $-\operatorname{div}(H(x) \nabla u)=1$ in $\Omega$ and $H(x)$ is a control. We introduce the functional $J_{\Omega}(H)=|\Omega|^{-1} \int_{\Omega} u(x) d x$ and show using a symmetrization argument that if the distribution function of $H$ is fixed, then $J_{\Omega}(H)$ is largest when $\Omega$ is a ball and $H$ is radial and decreasing on radii.
\end{abstract}

0. Introduction. In [4], Murat and Tartar considered the following problem: Let $B$ be a ball in $\mathbf{R}^{n}$ and let $u(x)$ satisfy

$$
-\operatorname{div}(H(x) \nabla u)=1 \quad \text { in } B, \quad u=0 \quad \text { on } \partial B,
$$

where $H(x)$ is a control variable in a class $\sharp$,

$$
H=\left\{H \in L^{\infty}(B): \alpha \leq H(x) \leq \beta, \int_{B} H d x=\gamma\right\},
$$

and $\alpha, \beta, \gamma$ are fixed positive constants. Determine the control $H_{0}(x)$ which maximizes

$$
J_{B}(H)=\frac{1}{|B|} \int_{B} u(x) d x
$$

over the class $H$. They find that $H_{0}(x)=\beta \chi_{B_{\rho}}+\alpha \chi_{B / B_{\rho}}$, where $B_{\rho}$ is a ball of radius $\rho$, concentric with $B$, and $\chi_{E}$ is the characteristic function of the set $E$.

In this paper we extend the class of controls $\sharp$ to be

$$
\mathscr{H}_{1}=\left\{H \in L^{\infty}(B), \alpha(x) \leq H(x) \leq \beta(x), \int_{B} H d x=\gamma\right\},
$$

where $\alpha$ and $\beta$ are fixed bounded functions which are radial, decreasing on radii, and satisfy $0<\alpha_{0} \leq \alpha(x) \leq \beta(x)$ a.e. in $B$. We find that the unique optimal control has the form

$$
H_{0}(x)=\beta(x) \chi_{B_{\rho}}+\alpha(x) \chi_{B / B_{\rho}} .
$$

While Murat and Tartar deduce their result as an application of homogenization, we employ a comparison theorem (Theorem 1.1 below) for solutions of $(0.1)$ involving rearrangement of the control $H$. This result enables us to reduce $\mathscr{H}_{1}$ to consist of radial functions, in which case the characterization of the optimal control is straightforward.

The comparison theorem has a long history dating back to 1856 when Saint Venant [6] conjectured the result in the case that $H$ is constant. A proof of the theorem in this case was obtained by Pólya in 1948 [5].

Received by the editors September 3, 1985.

1980 Mathematics Subject Classification (1985 Revision). Primary 49B22, 35J25; Secondary 35B05. 
1. Main results. If $H$ is a positive function in $L^{\infty}(\Omega), H \geq \alpha>0$, equation (0.1) has a unique weak solution in the Sobolev space $H_{0}^{1}(\Omega)$. This solution $u$ is characterized by the identity

$$
\int_{\Omega} H \nabla u \cdot \nabla \zeta d x=\int_{\Omega} \varsigma d x \quad \forall \zeta \in C_{0}^{1}(\Omega) .
$$

It is known [2] that the solution $u$ is actually locally Hölder continuous.

If $u \in H_{0}^{1}(\Omega)$ is the weak solution of $(0.1)$, we define

$$
J_{\Omega}(H)=\frac{1}{|\Omega|} \int_{\Omega} u d x
$$

where $|\Omega|$ denotes the measure of $\Omega$. We will frequently drop the domain dependence in denoting $J$ if there is no danger of ambiguity.

Suppose $\Omega$ is a bounded domain and let $f$ be a positive measurable function on $\Omega$. We denote the decreasing rearrangement of $f$ by $f^{*}$. Further, if $B$ is the ball centered at the origin such that $|B|=|\Omega|$, the symmetric decreasing rearrangement of $f$ is the function $f^{\#}$ defined on $B$ by the formula

$$
f^{\#}(x)=f^{*}\left(C_{n}|x|^{n}\right), \quad x \in B,
$$

where $C_{n}=\pi^{n / 2} / \Gamma(1+n / 2)$ is the measure of the unit ball in $\mathbf{R}^{n}$. We shall also need the following inequality of Hardy and Littlewood [3]. If $f$ and $g$ re nonnegative on $\Omega$, then

$$
\int_{\Omega} f g d x \leq \int_{0}^{|\Omega|} f^{*}(s) g^{*}(s) d s .
$$

THEOREM 1.1. Let $\Omega$ be a bounded domain in $\mathbf{R}^{n}(n \geq 1)$ and $H$ be a measurable function on $\Omega$ so $\alpha \leq H \leq \beta$ for positive constants $\alpha$ and $\beta$. If $H^{\#}$ is the symmetric decreasing rearrangement of $H$ on a ball $B \subset \mathbf{R}^{n}$, then

$$
J_{\Omega}(H) \leq J_{B}\left(H^{\#}\right) .
$$

Our proof of Theorem 1.1 is modelled after the argument of Talenti [8]. We note, however, that since our functional $J$ is lower semicontinuous [1], we can avoid the geometric measure theory in $[8]$ and reduce to the case $H \in C^{\infty}(\Omega)$.

PROOF OF THEOREM 1.1. If $n=1$, the theorem is easily proven so we take $n>1$. Let $R$ denote the radius of $B$, so $C_{n} R^{n}=|B|=|\Omega|$. Let $u \in H_{0}^{1}(\Omega)$ and $v \in H_{0}^{1}(B)$ be the weak solutions of

$$
\begin{gathered}
-\operatorname{div}(H \nabla u)=1 \quad \text { in } \Omega, \\
-\operatorname{div}\left(H^{\#} \nabla v\right)=1 \quad \text { in } B, \quad v=0 \text { on } \partial \Omega, \\
\text { on } \partial B .
\end{gathered}
$$

Note that since $H^{\#}$ is radial, $v$ is radial and we have

$$
\int_{B} v d x=\frac{C_{n}}{n} \int_{0}^{R} \frac{r^{n+1}}{H^{\#}(r)} d r=\frac{1}{n^{2} C_{n}^{2 / n}} \int_{0}^{|\Omega|} \frac{s^{2 / n}}{H^{*}(s)} d s .
$$

To obtain the estimate (1.4), we assume at first that $H \in C^{\infty}(\Omega)$. This restriction will be removed later by a passage to the limit. Since $H \in C^{\infty}(\Omega), u \in C^{\infty}(\Omega)$ (see [2, p. 109]). By the maximum principle, $u>0$ in $\Omega$, and if $\mu(\lambda)=|\{u>\lambda\}|$ is the distibution function of $u$, then $\mu$ is strictly decreasing, so $u^{*}=\mu^{-1}$ on the range 
of $\mu$. If $\lambda>0$ is not a critical value of $u$, then the open set $\{u>\lambda\}$ is bounded by the smooth compact manifold $\{u=\lambda\}$ which is contained in $\Omega$. We denote by $d \sigma$ the surface element on this surface. Note that by Sard's Theorem [7], the set of critical values of $u$ has measure zero.

If $\lambda>0$ is not a critical value of $u$, we may integrate both sides of (1.5) over the set $\{u>\lambda\}$ and apply the divergence theorem to obtain

$$
\mu(\lambda)=\int_{u>\lambda} d x=-\int_{u>\lambda} \operatorname{div}(H \nabla u) d x=-\int_{u=\lambda} H \nabla u \cdot n d \sigma,
$$

where $n$ is the outward unit normal to $\{u=\lambda\}$. Since $n=-\nabla u /|\nabla u|$, we see that

$$
\mu(\lambda)=\int_{u=\lambda} H|\nabla u| d \sigma .
$$

We now apply the isoperimetric inequality to estimate the surface area of $\{u=\lambda\}$. Since this set bounds a region of measure $\mu(\lambda)$, we find that

$$
\int_{u=\lambda} d \sigma \geq n C_{n}^{1 / n} \mu(\lambda)^{1-1 / n}
$$

Note that the boundary condition (1.5) is essential to this estimate.

Using the Cauchy-Schwarz inequality, we get

$$
\int_{u=\lambda} d \sigma \leq\left(\int_{u=\lambda} H|\nabla u| d \sigma\right)^{1 / 2}\left(\int_{u=\lambda} \frac{1}{H} \frac{1}{|\nabla u|} d \sigma\right)^{1 / 2} .
$$

Inequalities (1.8), (1.9), and (1.10) yield

$$
n^{2} C_{n}^{2 / n} \mu(\lambda)^{1-2 / n} \leq \int_{u=\lambda} \frac{1}{H} \frac{1}{|\nabla u|} d \sigma .
$$

Integrating both sides of (1.11) between 0 and $t$, we obtain

$$
n^{2} C_{n}^{2 / n} \int_{0}^{t} \mu(\lambda)^{1-2 / n} d \lambda \leq \int_{0}^{t} \int_{u=\lambda} \frac{1}{H} \frac{1}{|\nabla u|} d \sigma d t .
$$

But $(1 /|\nabla u|) d \sigma d \lambda$ is the volume element in $\Omega$, so if we apply the Hardy-Littlewood inequality (1.3), we see that the right-hand side of (1.12) may be estimated as follows:

$$
\begin{aligned}
\int_{0}^{t} \int_{u=\lambda} \frac{1}{H} \frac{1}{|\nabla u|} d \sigma d \lambda & =\int_{u<t} \frac{1}{H} d x \leq \int_{0}^{|\Omega|}\left(\frac{1}{H}\right)^{*}(w) \chi_{\{u<t\}}^{*}(w) d w \\
& =\int_{0}^{|\Omega|-\mu(t)}\left(\frac{1}{H}\right)^{*}(w) d w .
\end{aligned}
$$

Thus

$$
n^{2} C_{n}^{2 / n} \int_{0}^{t} \mu(\lambda)^{1-2 / n} d \lambda \leq \int_{0}^{|\Omega|-\mu(t)}\left(\frac{1}{H}\right)^{*}(w) d w .
$$

Making the change of variables $s=\mu(t)$ in (1.13), we find that for $0 \leq s \leq|\Omega|$,

$$
n^{2} C_{n}^{2 / n} \int_{0}^{u^{*}(s)} \mu(\lambda)^{1-2 / n} d \lambda \leq \int_{0}^{|\Omega|-s}\left(\frac{1}{H}\right)^{*}(w) d w .
$$


Next we multiply both sides of $(1.14)$ by $s^{2 / n-1}$ and integrate from 0 to $|\Omega|$ to obtain

$$
\begin{aligned}
n^{2} C_{n}^{2 / n} \int_{0}^{|\Omega|} s^{2 / n-1} \int_{0}^{u^{*}(s)} \mu(\lambda)^{1-2 / n} d \lambda d s \\
\quad \leq \int_{0}^{|\Omega|} s^{2 / n-1} \int_{0}^{|\Omega|-s}\left(\frac{1}{H}\right)^{*}(w) d w d s \\
\quad=\frac{n}{2} \int_{0}^{|\Omega|} s^{2 / n}\left(\frac{1}{H}\right)^{*}(|\Omega|-s) d s \\
\quad=\frac{n}{2} \int_{0}^{|\Omega|} \frac{s^{2 / n}}{H^{*}(s)} d s=\frac{n^{3}}{2} C_{n}^{2 / n} \int_{B} v d x \quad \text { (by (1.7)). }
\end{aligned}
$$

Note that we have used $(1 / H)^{*}(|\Omega|-s)=1 / H^{*}(s)$, since the two functions are increasing with the same distribution function.

Canceling constants in the preceding inequality and changing the order of integration yields

$$
\begin{aligned}
\int_{B} v d x & \geq \frac{2}{n} \int_{0}^{|\Omega|} s^{2 / n-1} \int_{0}^{u^{*}(s)} \mu(\lambda)^{1-2 / n} d \lambda d s \\
& =\frac{2}{n} \int_{0}^{u^{*}(0)} \int_{0}^{\mu(\lambda)} s^{2 / n-1} \mu(\lambda)^{1-2 / n} d s d \lambda \\
& =\int_{0}^{u^{*}(0)} \mu(\lambda) d \lambda=\int_{0}^{|\Omega|} u^{*}(s) d s=\int_{\Omega} u d x
\end{aligned}
$$

which was to be shown.

To deduce the theorem for measurable $H$, we use the fact that the functional $H \mapsto J(H)$ is lower semicontinuous with respect to weak* convergence in $L^{\infty}(\Omega)$ (see [1, Theorem 2.1]). Let $H \in L^{\infty}(\Omega)$ and $a \leq H \leq b$ for positive constants $a$ and $b$. Choose a sequence $H_{m} \in C^{\infty}(\Omega)$ so

(a) $H_{m} \rightarrow H$ in $L^{2}(\Omega)$ and a.e.,

(b) $a \leq H_{m} \leq b$ for all $m$.

Then $H_{m} \rightarrow H$ weak $^{*}$ in $L^{\infty}$. Further, it follows from (1.3) that $H_{m}^{*} \rightarrow H^{*}$ in $L^{2}(0,|\Omega|)$, so, by passing to a subsequence if necessary, we may assume that

(c) $H_{m}^{*} \rightarrow H^{*}$ a.e. on $(0,|\Omega|)$.

Now let $u_{m} \in H_{0}^{1}(\Omega)$ and $v_{m} \in H_{0}^{1}(B)$ be the weak solutions to

$$
-\operatorname{div}\left(H_{m} \nabla u_{m}\right)=1 \text { and }-\operatorname{div}\left(H_{m}^{\#} \nabla v_{m}\right)=1 .
$$

We obtain, using the lower semicontinuity of $J$ and the dominated convergence theorem,

$$
\begin{aligned}
\int_{\Omega} u d x & \leq \liminf _{m \rightarrow \infty} \int_{\Omega} u_{m} d x \\
& \leq \liminf _{m \rightarrow \infty} \int_{B} v_{m} d x \quad\left(\text { since } H_{m} \in C^{\infty}(\Omega)\right) \\
& =\liminf _{m \rightarrow \infty} \frac{C_{n}^{-2 / n}}{n^{2}} \int_{0}^{|\Omega|} \frac{s^{2 / n}}{H_{m}^{*}(s)} d s \quad(\text { by }(1.7)) \\
& =\frac{C_{n}^{-2 / n}}{n^{2}} \int_{0}^{|\Omega|} \frac{s^{2 / n}}{H^{*}(s)} d s=\int_{B} v d x .
\end{aligned}
$$


Thus $J_{\Omega}(H) \leq J_{B}\left(H^{\#}\right)$ as was to be shown.

REMARK. If we take $s=0$ in (1.14) we obtain

$$
n^{2} C_{n}^{2 / n} \int_{0}^{u^{*}(0)} \mu(\lambda)^{1-2 / n} d \lambda \leq \int_{0}^{|\Omega|}\left(\frac{1}{H}\right)^{*} d x=\int_{\Omega} \frac{1}{H} d x .
$$

This inequality is particularly simple if $n=2$, in which case it beccomes

$$
\|u\|_{\infty}=u^{*}(0) \leq \frac{1}{4 \pi} \int_{\Omega} \frac{1}{H} d x .
$$

THEOREM 1.2. Let $B$ be a ball centered at the origin in $\mathbf{R}^{n}$ of radius $R$. Let $\alpha, \beta$ be radial functions in $L^{\infty}(B)$ which are decreasing on radii, and which satisfy ess inf $\alpha(x)>0$ and $\alpha(x) \leq \beta(x)$ a.e. Let $\gamma$ be a constant so

$$
\int_{B} \alpha d x \leq \gamma \leq \int_{B} \beta d x
$$

Let $H_{1}$ denote the subset of $L^{\infty}(B)$ defined in (0.2). Then there exists a unique $\rho$, $0 \leq \rho \leq R$, so if $H_{0}=\beta \chi_{B_{\rho}}+\alpha \chi_{B / B_{\rho}}$, where $B_{\rho}$ denotes the ball centered at the origin of radius $\rho$, then $H_{0} \in H_{1}$ and

$$
J_{B}(H) \leq J_{B}\left(H_{0}\right) \quad \text { for all } H \in \mathscr{H}_{1}
$$

with equality if and only if $H=H_{0}$.

Proof. Let $r=|x|$ denote the radial variable in $\Omega$. We observe that there exists a unique $\rho \in[0, R]$ so the function $H_{0}(x)=\beta(x) \chi_{B_{\rho}}(x)+\alpha(x) \chi_{B / B_{\rho}}(x)$ is in $\varkappa_{1}$. Indeed, the function

$$
\phi(\rho)=\int_{B_{\rho}} \beta d x+\int_{B / B_{\rho}} \alpha d x, \quad 0 \leq \rho \leq R,
$$

is a strictly increasing absolutely continuous function, and since $\phi(0) \leq \gamma \leq \phi(R)$ by $(1.15)$, there exists a unique $\rho$ so $\phi(\rho)=\gamma$.

Now let $H \in \mathscr{H}_{1}$. Since $\alpha$ and $\beta$ are radial and decreasing, we have $\alpha=\alpha^{\#}$ and $\beta=\beta^{\#}$ a.e. in $B$, and one verifies easily that since $\alpha \leq H \leq \beta$, we have $\alpha^{\#} \leq$ $H^{\#} \leq \beta^{\#}$. Since $\int_{B} H d x=\int_{B} H^{\#} d x$, we have $H^{\#} \in H_{1}$, and by Theorem $1 . \overline{1}$, $J(H) \leq J\left(H^{\#}\right)$. This observation enables us to reduce our consideration to the case that $H=H(r)$ is a radial function which is decreasing for $0 \leq r \leq R$. If $H \in \mathscr{H}_{1}$ is such a function, we show that $J(H) \leq J\left(H_{0}\right)$ with equality only if $H=H_{0}$. This will complete the proof of Theorem 1.2.

Thus assume that $H \in \mathscr{H}_{1}$ is radial and decreasing. Using (1.7) we calculate that

$$
J\left(H_{0}\right)-J(H)=\frac{1}{n R^{n}} \int_{0}^{R} r^{n+1} \frac{\left(H-H_{0}\right)}{H_{0} H} d r .
$$

Since $\int_{0}^{R} H r^{n-1} d r=\int_{0}^{R} H_{0} r^{n-1} d r$, we may rewrite this equation as

$$
J\left(H_{0}\right)-J(H)=\frac{1}{n R^{n}} \int_{0}^{R} r^{n-1}\left(H-H_{0}\right)\left(\frac{r^{2}}{H H_{0}}-c\right) d r,
$$

where $c$ is any constant. Since $r^{2} / H H_{0}$ is increasing on $(0, R)$, we may choose $c$ so that

$$
\lim _{r \rightarrow \rho^{-}} \frac{r^{2}}{H(r) H_{0}(r)} \leq c \leq \lim _{r \rightarrow \rho^{+}} \frac{r^{2}}{H(r) H_{0}(r)} .
$$


Then since $\left(H-H_{0}\right)(r) \leq 0$ if $r<\rho$ and $\left(H-H_{0}\right)(r) \geq 0$ if $r>\rho$, we find that the integrand in (1.17) is nonnegative, so $J\left(H_{0}\right) \geq J(H)$.

If $J(H)=J\left(H_{0}\right)$ for some $H \neq H_{0}$, then we see from (1.17) that $H H_{0}=r^{2} / c$ on the set where $H \neq H_{0}$. Thus $H H_{0}$ is strictly increasing on a set of positive measure, which is a contradiction. Thus $J(H)=J\left(H_{0}\right)$ implies $H=H_{0}$ a.e., and the proof is complete.

\section{REFERENCES}

1. J. Cea and K. Malanowski, An example of a max-min problem in partial differential equations, SIAM J. Control Optim. 8 (1970), 305-316.

2. D. Gilbarg and N. Trudinger, Elliptic partial differential equations of second order, Springer-Verlag, Berlin, 1983.

3. G. H. Hardy, J. F. Littlewood, and G. Pólya, Inequalities, Cambridge Univ. Press, Cambridge, 1967.

4. F. Murat and L. Tartar, Calcul de variations et homogenization, Cours de l'Ecole d'Ete d'Analyse Numerique, 1984.

5. G. Pólya, Torsional rigidity, principal frequencey, electrostatic capacity and symmetrization, Quart. Appl. Math. 6 (1948), 267-277.

6. B. de Saint-Venant, Memoire sur la torsion des prismes, Memoires Presentes par Divers Savants a l'Academie des Sciences, t. 14, 1856, pp. 233-560.

7. S. Sternberg, Lectures in differential geometry, Prentice-Hall, Englewod Cliffs, N.J., 1964.

8. G. Talenti, Elliptic equations and rearrangements, Ann. Scuola Norm. Sup. Pisa Cl. Sci. (4) 3 (1976), 697-718.

Department of Mathematics, Lehigh University, Bethlehem, Pennsylvania 18015 (Current address of Charles Voas)

Current address (Daniel Yaniro): AT\&T Bell Laboratories, Crawford Corner Road, Holmdel, New Jersey 07733 